\title{
Line-dependent veiling in very active classical T Tauri stars
}

\author{
A. C. S. Rei ${ }^{1,2}$, P. P. Petrov ${ }^{3}$, and J. F. Gameiro ${ }^{1,2}$ \\ ${ }^{1}$ Instituto de Astrofísica e Ciências do Espaço, CAUP, Rua das Estrelas, 4150-762 Porto, Portugal \\ e-mail: ana.rei@astro.up.pt \\ 2 Departamento de Física e Astronomia, Faculdade de Ciências, Universidade do Porto, Rua do Campo Alegre, 4150-762 Porto, \\ Portugal \\ ${ }^{3}$ Crimean Astrophysical Observatory of Russian Academy of Sciences, p/o Nauchny, 298409, Crimea
}

Received 26 June 2017 / Accepted 22 November 2017

\begin{abstract}
Context. The T Tauri stars with active accretion disks show veiled photospheric spectra. This is supposedly due to non-photospheric continuum radiated by hot spots beneath the accretion shocks at stellar surface and/or chromospheric emission lines radiated by the post-shocked gas. The amount of veiling is often considered as a measure of the mass-accretion rate.

Aims. We analysed high-resolution photospheric spectra of accreting T Tauri stars LkH $\alpha$ 321, V1331 Cyg, and AS 353A with the aim of clarifying the nature of the line-dependent veiling. Each of these objects shows a strong emission line spectrum and powerful wind features indicating high rates of accretion and mass loss.

Methods. Equivalent widths of hundreds of weak photospheric lines were measured in the observed spectra of high quality and compared with those in synthetic spectra of appropriate models of stellar atmospheres.

Results. The photospheric spectra of the three T Tauri stars are highly veiled. We found that the veiling is strongly line-dependent: larger in stronger photospheric lines and weak or absent in the weakest ones. No dependence of veiling on excitation potential within 0 to $5 \mathrm{eV}$ was found. Different physical processes responsible for these unusual veiling effects are discussed in the framework of the magnetospheric accretion model.

Conclusions. The observed veiling has two origins: (1) an abnormal structure of stellar atmosphere heated up by the accreting matter, and (2) a non-photospheric continuum radiated by a hot spot with temperature lower than $10000 \mathrm{~K}$. The true level of the veiling continuum can be derived by measuring the weakest photospheric lines with equivalent widths down to $\approx 10 \mathrm{~m} \AA$. A limited spectral resolution and/or low signal-to-noise ratio results in overestimation of the veiling continuum. In the three very active stars, the veiling continuum is a minor contributor to the observed veiling, while the major contribution comes from the line-dependent veiling.
\end{abstract}

Key words. stars: activity - stars: pre-main sequence - stars: variables: T Tauri, Herbig Ae/Be - stars: individual: LkH $\alpha 321$ stars: individual: V1331 Cyg - stars: individual: AS 353A

\section{Introduction}

The T Tauri stars are late-type stars at the pre-main sequence (PMS) phase of evolution. These stars are in fact star-disk systems and their spectra show several features associated with the interaction between star and disk. There are two classes of $\mathrm{T}$ Tauri stars, based on the degree of activity of the star-disk system: classical T Tauri stars (cTTS) and weak-line T Tauri stars (wTTS). The rich emission spectrum of cTTS is related to magnetospheric accretion of matter from the inner disk regions onto the star. Yet in the early studies of cTTS it was found that the strength of the photospheric lines is reduced as compared to a main-sequence (MS) star of the corresponding spectral type. Joy (1949) noted that this may be due to emission within the lines and due to veiling of the spectrum by an overlying continuous radiation.

In the concept of magnetospheric accretion, the veiling continuum is attributed to a shock-heated gas (a hot spot) at the stellar surface below the accretion stream. The veiling was often considered as a measure of accretion rate (e.g. Basri \& Batalha 1990; Hartigan et al. 1991, 1995; Valenti et al. 1993; Calvet et al. 2004) along with other indicators, like the luminosity in $\mathrm{H} \alpha$ and other emission lines (e.g. Muzerolle et al. 1998;
Herczeg \& Hillenbrand 2008; Dahm 2008; Fang et al. 2009; Rigliaco et al. 2012; Mendigutía et al. 2015).

As more data were accumulated, some doubts appeared about the interpretation of the veiling origin. The expected correlation between the veiling in the optical spectrum and the brightness of the star was not found in some cTTS. For example, in RW Aur A the veiling is highly variable from night to night. However, no correlation has been found with the stellar brightness in the $V$ band from the photometric and spectroscopic monitoring of the star (Petrov et al. 2001). No rotational modulation of the veiling was reported for the star.

Gahm et al. (2008) showed that in RU Lup and S CrA SE large veiling factors correlate only weakly with brightness. In the case of S CrA SE a narrow emission core appears at the bottom of photospheric absorptions at moments of enhanced accretion. Furthermore, when the veiling is measured carefully in every single spectral line within a narrow wavelength range, it turns out that the veiling depends on the line strength in a template of the corresponding spectral type. Stronger lines can be veiled considerably, while the weakest lines remain about normal for the spectral type. So far, line-dependent veiling has been found in DR Tau (Petrov et al. 2011) and V1331 Cyg (Petrov et al. 2014). A similar effect was noticed earlier in 


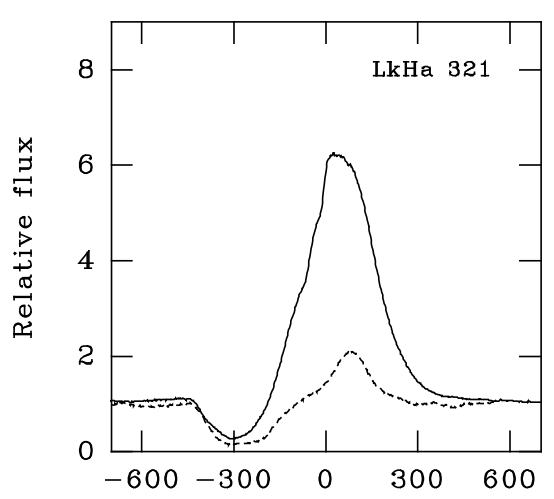

Radial velocity, $\mathrm{km} / \mathrm{s}$
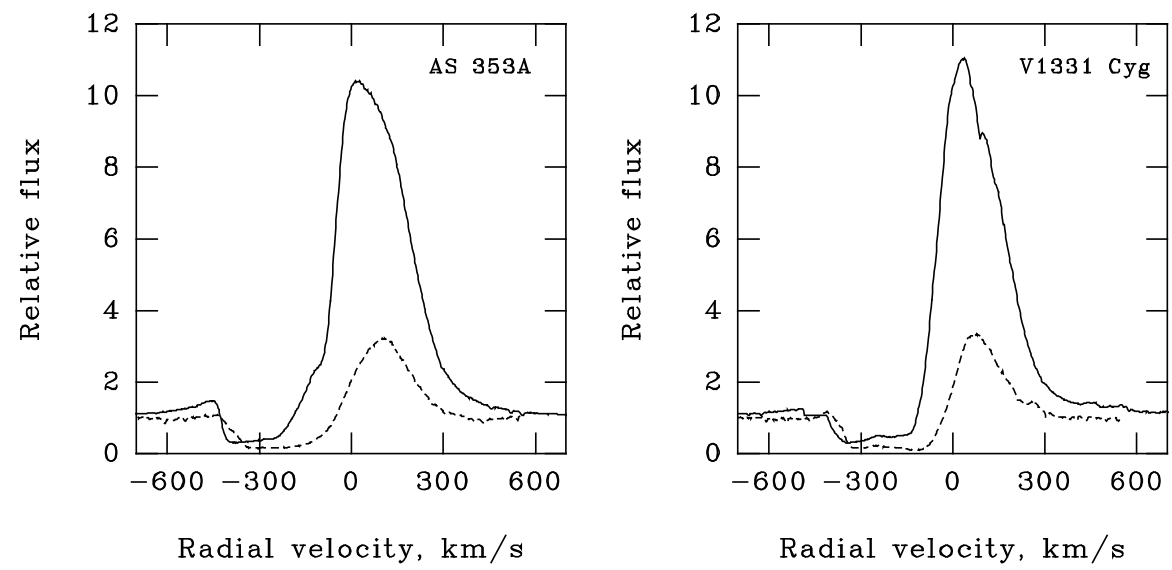

Fig. 1. $\mathrm{H} \alpha$ (solid line) and $\mathrm{H} \beta$ (dashed line) profiles.

DI Cep (Gameiro et al. 2006). Line-dependent veiling was found in spectra of EX Lup in quiescent state, when the accretion rate was relatively low (Sicilia-Aguilar et al. 2015). The authors concluded that photospheric lines in EX Lup are veiled by the broad emission lines from the extended pre-shock material.

In order to clarify the nature of line-dependent veiling, we undertake detailed inspection of photospheric lines in cTTS with high accretion rates. In this work we investigate the veiling effect in spectra of three cTTS: LkH $\alpha 321$ (=V1980 Cyg), AS 353A (=V1352 Aql), and V1313 Cyg.

\section{Observational data}

In this research we use spectra obtained by George Herbig with the High Resolution Echelle Spectrometer (HIRES) at Keck- $1^{1}$ (Vogt et al. 1994). We estimated a spectral resolution of $R \sim 50000$ near $6300 \AA$ from the weakest telluric lines in this region.

The spectra were obtained on 2002 December 16 ( $\mathrm{LkH} \alpha$ 321), 2003 July 6 (AS 353A), and 2004 July 24 (V1331 Cyg). The wavelength coverage was 4350-6750 $\AA$ with some gaps between spectral orders in the region $>5000 \AA$. One more spectrum of V1331 Cyg was taken on 2007 November 23 with the same spectrograph and a mosaic of three CCDs covering the spectral range 4750-8690 $\AA$. In the spectra of $\mathrm{LkH} \alpha 321$ and V1331 Cyg, the signal-to-noise (S/N) ratio per resolution element is 170 at $5000 \AA$ and rises to 400 at $6500 \AA$. The spectrum of AS 353A has a S/N of 200 at $5000 \AA$ and 350 at $6500 \AA$.

The spectra analysed belong to three cTTS with high accretion rates, whose stellar parameters are difficult to estimate. A summary of previous and current estimations of some stellar parameters for these stars can be found in Table 1. The estimations of spectral type, effective temperature $\left(T_{\text {eff }}\right)$, projected rotational velocity $(v \sin i)$, radial velocity $\left(v_{\mathrm{rad}}\right)$, and equivalent widths of the $\mathrm{H} \alpha(\mathrm{EW}(\mathrm{H} \alpha))$ and He I $5875 \AA(\mathrm{EW}(\mathrm{HeI}))$ lines for each star are described in Sect. 3.

Utrecht Echelle Spectrograph (UES) spectra of each star were also available, from observations done in November of 1998. The UES spectra have a similar resolution to those of HIRES, but with a $S / N<100$. Although the $\mathrm{S} / \mathrm{N}$ is not high

\footnotetext{
1 The W. M. Keck Observatory is operated as a scientific partnership between the California Institute of Technology, the University of California, and the National Aeronautics and Space Administration. The Observatory was made possible by the generous financial support of the W. M. Keck Foundation.
}

enough to perform the same type of study done with the HIRES spectra, it is possible to check for a change in radial velocity of the stars from spectra taken in different epochs.

The three objects show strong emission line spectra, powerful wind features, and highly veiled photospheric spectra. Typically, in spectra of cTTS the emission lines of neutral and ionized metals exhibit a composite profile: a broad component formed in the accretion funnel and a narrow component consistent with an origin in the post-shocked gas near the stellar surface (e.g. Beristain et al. 1998). This is the case of $\mathrm{LkH} \alpha 321$ and AS $353 \mathrm{~A}$, where the broad component is dominant with full width at half maximum $(F W H M) \approx 140 \mathrm{~km} \mathrm{~s}^{-1}$. On the contrary, in V1331 Cyg the numerous emission lines of metals show only a single moderately broad profile with $F W H M=40-60 \mathrm{~km} \mathrm{~s}^{-1}$ (see Sect. 7.1).

The most prominent lines are the hydrogen Balmer and Paschen lines, the CaII, NaI D, and O I $8446 \AA$ lines with $F W H M \approx 200 \mathrm{~km} \mathrm{~s}^{-1}$. These broad lines are supposedly formed in the high velocity infalling gas at the base of the accretion column, before the shock (pre-shocked gas), within the stellar magnetosphere. The $\mathrm{P}$ Cyg type of the profiles of $\mathrm{H} \alpha$ and $\mathrm{H} \beta$ lines indicates an extensive mass-loss (Fig. 1).

Figure 2 compares two spectral fragments of the stars to show the differences in strength and width between the photospheric and emission lines. In all three objects the narrow forbidden emissions of [O I] 6300.3 and $6363.7 \AA$ are present.

\section{Data analysis}

Template spectra of non-active main-sequence (MS) stars, wTTS, or synthetic spectra are needed to perform the analysis of cTTS. In this investigation we analyse rather weak spectral lines, with equivalent width (EW) of about $10 \mathrm{~m} \AA$, so we need high-resolution, high $\mathrm{S} / \mathrm{N}$ spectra of template stars of different spectral type. In order to minimize the errors of measurements, we prefer to use a grid of synthetic spectra. This way we avoid possible spectral peculiarities from the real stars that could affect our results.

\subsection{Template synthetic spectra}

The synthetic spectra were produced by the software package Spectroscopy Made Easy (SME; Valenti \& Piskunov 1996). It was initially used to confirm the spectral type of each cTTS, with the estimation of $T_{\text {eff }}$, as well as for the determination of $v \sin i$ and $v_{\text {rad. }}$. 
Table 1. Stellar parameters.

\begin{tabular}{ccccccccccc}
\hline \hline Star & Sp.type & $\begin{array}{c}T_{\text {eff }} \\
(\mathrm{K})\end{array}$ & $\begin{array}{c}\log L_{*} \\
\left(L_{\odot}\right)\end{array}$ & $\begin{array}{c}M_{*}^{d} \\
\left(M_{\odot}\right)\end{array}$ & $\begin{array}{c}R_{*}^{d} \\
\left(R_{\odot}\right)\end{array}$ & $\begin{array}{c}\log g^{d} \\
(\mathrm{dex})\end{array}$ & $\begin{array}{c}v \sin i \\
\left(\mathrm{~km} \mathrm{~s}^{-1}\right)\end{array}$ & $\begin{array}{c}v_{\text {rad }}(\mathrm{HIRES}) \\
\left(\mathrm{km} \mathrm{s}^{-1}\right)\end{array}$ & $\begin{array}{c}\mathrm{EW}(\mathrm{H} \alpha) \\
(\AA)\end{array}$ & $\begin{array}{c}\mathrm{EW}(\mathrm{HeI}) \\
(\AA)\end{array}$ \\
\hline LkH $\alpha$ 321 & G5-G7 & $5500-5250$ & $1.42^{a}$ & 2.9 & 5.3 & 3.5 & 32 & $-16.0 \pm 0.5$ & 30 & 0.08 \\
V1331 Cyg & G7-K1 & $5250-5000$ & $1.32^{b}$ & 2.9 & 5.5 & 3.5 & $<6$ & $-15.0 \pm 0.3$ & 53 & 0.22 \\
AS 353A & K0-K1 & $5100-4900$ & $0.4^{c}$ & 1.6 & 2.1 & 4.0 & $<6$ & $-10.4 \pm 0.2$ & 58 & 1.30 \\
\hline
\end{tabular}

Notes. Sp.type, $T_{\text {eff }}, v \sin i, v_{\text {rad }}, \mathrm{EW}(\mathrm{H} \alpha)$, and EW(HeI) are from this paper. ${ }^{(a)}$ Cohen \& Kuhi (1979), ${ }^{(b)}$ Tokunaga et al. (2004), ${ }^{(c)}$ Hamann \& Persson (1992), ${ }^{(d)}$ Obtained from $T_{\text {eff }}$ and $L_{*}$ and using the PMS models by Siess et al. (2000).

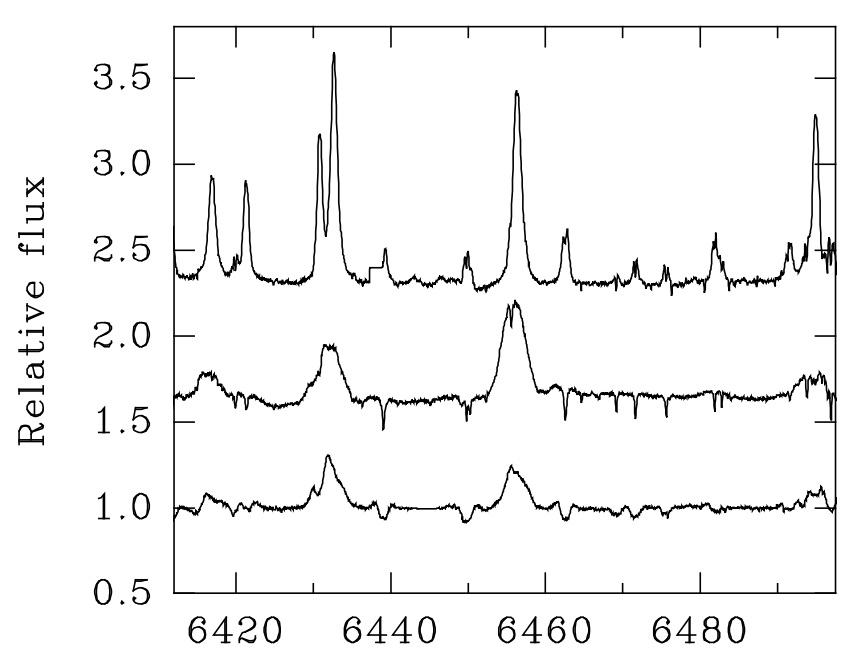

Wavelength, A

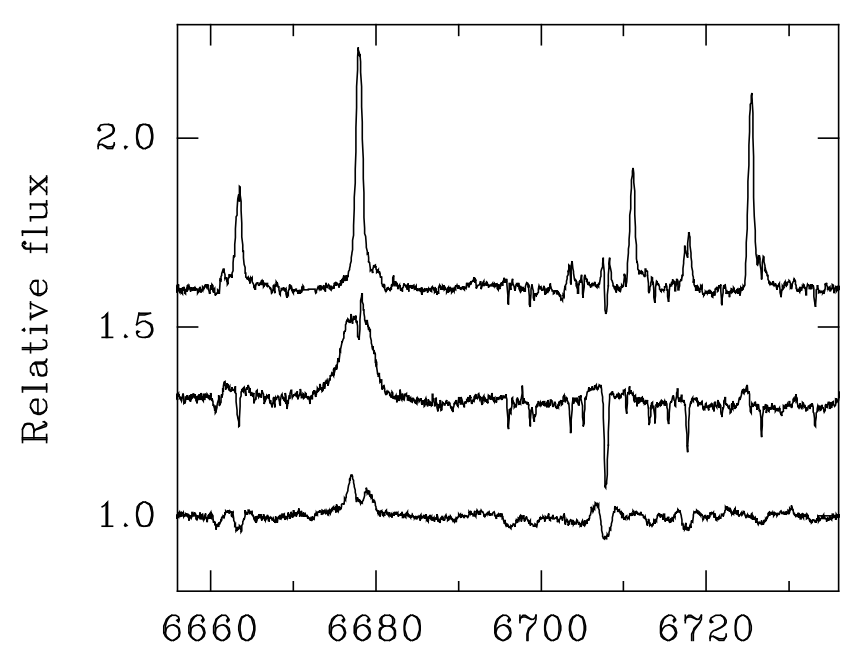

Wavelength, A

Fig. 2. Fragments of spectra: $\mathrm{LkH} \alpha 321$ (lower), AS 353A (middle), and V1331 Cyg (upper).

Two sets of synthetic spectra with spectral resolution $R=50000$ were produced. The first set corresponds to a grid of narrow-band spectra with several values of stellar parameters, which was used in the determination of spectral type, as well as $v \sin i$ and $v_{\mathrm{rad}}$. Then, specific spectra for each star in a wider wavelength band between 4500 and $7000 \AA$ were produced. This second set of synthetic spectra corresponds to our templates and was used to measure EWs of hundreds of spectral lines for comparison with the same lines in the spectra of cTTS.

The spectral type determination, described in detail in Sect. 3.2, allowed us to gather information mainly about $T_{\text {eff }}$. The usual criteria of surface gravity $(\log g)$ can hardly be used in the case of highly veiled spectra with strong lines in emission. Due to that we are assuming the value $\log g=3.75$ dex for these stars (see Sect. 3.2). We also assume a priori a solar-like metallicity $([\mathrm{M} / \mathrm{H}]=0.0)$.

Besides the mentioned stellar parameters, SME also needs input information about the microturbulence velocity $\left(v_{\text {mic }}\right)$ and macroturbulence velocity $\left(v_{\text {mac }}\right)$, as well as the instrumental resolution. For the $v_{\text {mic }}$ parameter we assume a value that is typical for cool stars and wTTS (e.g. Padgett 1996; Rojas et al. 2008; James et al. 2006; Taguchi et al. 2009): $v_{\text {mic }}=1.0 \mathrm{~km} \mathrm{~s}^{-1}$. For the $v_{\text {mac }}$ value we assume a value based on the $T_{\text {eff }}$ value, as reported by Valenti \& Fischer (2005). The three stars have spectral types between $\mathrm{K} 1$ and G5 corresponding to temperatures between 4900 and $5500 \mathrm{~K}$ for sub-giant stars (Table 1). For this range of temperatures we assume a value of $v_{\text {mac }}=2.8 \mathrm{~km} \mathrm{~s}^{-1}$, consonant to an average $T_{\text {eff }}=5000 \mathrm{~K}$.

To create the synthetic spectra we use the Kurucz grids of stellar atmospheric models, considering local thermodynamic equilibrium (LTE) conditions. The line list used by SME to create the synthetic spectra was extracted from the Vienna Atomic Line Database (VALD) database using the "Extract stellar" option (Ryabchikova et al. 2015). Since we measured several hundred spectral lines, we did not apply any corrections to the $\log g f$ and Van der Waals damping parameters.

\subsection{Spectral type determination}

The spectral types of our targets were determined by using a grid of synthetic spectra with several values of $T_{\text {eff }}$ and $\log g$. Determination of the spectral type of cTTS is hampered by the veiling effect. As will be shown below, the stronger lines are most affected by the veiling, therefore the use of strong lines in the spectral classification should be avoided. To perform this task we identified the less blended photospheric lines on several spectral fragments throughout the whole spectrum.

To find the value of $T_{\text {eff }}$ for each star, we identified several pairs of weak lines with ratios sensitive to temperature changes in the synthetic spectra grid. The synthetic spectra with the ratios of lines similar to those of the cTTS were chosen as the appropriate templates. Typically, the cTTS spectra drop in between two synthetic templates with $250 \mathrm{~K}$ difference. The pairs of photospheric lines used to determine the spectral type of each cTTS are listed in Table 2.

Due to the numerous emission lines and the veiling of photospheric lines, it is problematic to derive the $\log g$ parameter directly from the spectra of very active cTTS. It can be estimated from the grid of models of PMS stars (Siess et al. 2000) with input parameters $T_{\text {eff }}$ and $L_{*}$ (see Table 1). For the synthetic 
Table 2. Photospheric line pairs used in the spectral type determination.

\begin{tabular}{ccccccc}
\hline \hline \multirow{2}{*}{ Star } & & Line 1 & & & Line 2 & \\
& $\lambda(\AA)$ & Ele. & Exc. pot. $(\mathrm{eV})$ & $\lambda(\AA)$ & Ele. & Exc. pot. $(\mathrm{eV})$ \\
\hline \multirow{5}{*}{ LkH $\alpha$ 321 } & 6013.416 & Ti I & 1.07 & 6016.605 & Fe I & 3.55 \\
& 6039.729 & V I & 1.06 & 6041.950 & Fe I & 4.96 \\
& 6325.164 & Ti I & 0.02 & 6327.599 & Ni I & 1.68 \\
& 6469.193 & Fe I & 4.48 & 6471.662 & Ca I & 2.53 \\
\hline \multirow{5}{*}{ V1331 Cyg } & 5054.074 & Ti I & 2.68 & 5054.642 & Fe I & 3.64 \\
& 5289.269 & Ti I & 0.84 & 5289.817 & Y II & 1.03 \\
& 5295.312 & Fe I & 4.42 & 5295.776 & Ti I & 1.07 \\
& 5376.599 & Ti I & 0.00 & 5376.830 & Fe I & 4.29 \\
& 5384.630 & Ti I & 0.83 & 5385.575 & Fe I & 3.69 \\
& 5440.133 & V I & 2.61 & 5385.575 & Fe I & 3.69 \\
& 5460.499 & Ti I & 1.43 & 5441.339 & Fe I & 4.31 \\
& 5465.773 & Ti I & 0.05 & 5461.549 & Fe I & 4.45 \\
& 6111.070 & Ni I & 4.09 & 6111.651 & V I & 1.04 \\
& 6116.180 & Ni I & 4.27 & 6116.990 & Co I & 1.79 \\
& 6116.180 & Ni I & 4.27 & 6119.528 & V I & 1.06 \\
AS 353A & 6146.207 & Ti I & 1.87 & 6147.834 & Fe I & 4.08 \\
& 6151.617 & Fe I & 2.18 & 6152.292 & Si I & 5.96 \\
& 6154.225 & Na I & 2.10 & 6155.134 & Si I & 5.62 \\
& 6156.023 & Ca I & 2.52 & 6157.727 & Fe I & 4.08 \\
\hline
\end{tabular}

Notes. Some of the lines listed in this table are blends, particularly for the $\mathrm{LkH} \alpha 321$ spectrum.

Table 3. Template synthetic spectra parameters for each star.

\begin{tabular}{ccccccc}
\hline \hline $\begin{array}{c}\text { Template } \\
\text { for }\end{array}$ & $\begin{array}{c}T_{\text {eff }} \\
(\mathrm{K})\end{array}$ & $\begin{array}{c}\log g \\
(\mathrm{dex})\end{array}$ & $\begin{array}{c}{[\mathrm{M} / \mathrm{H}]} \\
(\mathrm{dex})\end{array}$ & $\begin{array}{c}v \sin i \\
\left(\mathrm{~km} \mathrm{~s}^{-1}\right)\end{array}$ & $\begin{array}{c}v_{\text {mic }} \\
\left(\mathrm{km} \mathrm{s}^{-1}\right)\end{array}$ & $\begin{array}{c}v_{\mathrm{mac}} \\
\left(\mathrm{km} \mathrm{s}^{-1}\right)\end{array}$ \\
\hline $\mathrm{LkH} \alpha 321$ & 5250 & 3.75 & 0.0 & 32 & 1.0 & 2.8 \\
V1331 Cyg & 5000 & 3.75 & 0.0 & 1 & 1.0 & 2.8 \\
AS 353A & 5000 & 3.75 & 0.0 & 1 & 1.0 & 2.8 \\
\hline
\end{tabular}

templates we adopted $\log g=3.75$ dex. A simple test with the templates showed that differences of 0.25 dex in this parameter are not critical for our analysis of equivalent widths.

For the determination of the $v \sin i$ value, artificial veiling must be added to the synthetic spectra in order to mimic the real spectra. Because the estimation of veiling also depends on the $v \sin i$ value, this process must be done in an iterative way, until the synthetic spectra lines have a good match with the real ones.

The information regarding the synthetic templates for each cTTS, based on the previous estimation of stellar parameters, is summarized in Table 3. Details on the individual spectrum of each star can be found in Sects. 4-6.

\subsection{Equivalent widths measurements}

Our study is based on the comparison of EWs of several hundred photospheric lines of cTTS with those of synthetic templates. To measure the EWs we used IRAF ${ }^{2}$. Due to the presence of strong veiling and emission in these stars' spectra, only lines that could be clearly identified with their counterparts in the

\footnotetext{
2 IRAF is distributed by the National Optical Astronomy Observatories, which are operated by the Association of Universities for Research in Astronomy, Inc., under cooperative agreement with the National Science Foundation.
}

synthetic templates were measured. Regions with broad emission were avoided. In total, we measured the EWs of more than 500 photospheric lines, except for $\mathrm{LkH} \alpha 321$, where many lines are blended because of the large $v \sin i$. We measured EWs of the less blended lines in $\mathrm{LkH} \alpha 321$ and in the corresponding template with the same $v \sin i$.

Although the precision of the measurements is limited by $\mathrm{S} / \mathrm{N}$, the main source of errors is the uncertainty in the local continuum level, caused by the line blends of both photospheric absorptions and broad emissions. We use the synthetic template to find the adjacent points of the continuum for each photospheric line, and select the spectral intervals where the broad emissions are relatively small, not exceeding a few percent above the continuum level. The relative error in the EW of photospheric lines was found to be within 5-12\%.

\subsection{Equivalent widths ratio as a measure of veiling}

Traditionally, on cTTS the veiling factor (VF) is expressed as $V F=E W($ template $) / E W($ tts $)-1$. In this expression it is assumed that $\mathrm{EW}(\mathrm{tts})$ is reduced by an additional (nonphotospheric) continuum, and the VF is a measure of this non-photospheric continuum in units of the photospheric continuum. Since we do not know a priori the nature of the veiling, 
we will use in the following analysis only the ratio of EWs: $\mathrm{EW}$ (template)/EW(tts). In the veiled spectrum of cTTS, for most of the photospheric lines this ratio is larger than unity, although for the weakest lines it may be less than unity because of the measurement errors. The error in spectral type may also result in some bias. In the optical spectrum of cTTS, the veiling usually rises towards the blue region, which indicates the presence of a hot continuum (Basri \& Batalha 1990; Calvet \& Gullbring 1998). In this study we also analyse how the veiling changes with wavelength.

We used real spectra of the Sun and a G7IV star (HD190248) to evaluate how large the scatter of the EW(synthetic)/EW(real) is along the spectrum. The Sun spectrum $^{3}$ was obtained by High Accuracy Radial velocity Planet Searcher (HARPS) and has a $S / N>500$ (Blanco-Cuaresma et al. 2014). The G7IV star spectrum was extracted from the ESO Archive. We assigned a synthetic template with $T_{\text {eff }}=5000 \mathrm{~K}$ and $\log g=3.75$ dex to the G7IV star and a template with $T_{\text {eff }}=5750 \mathrm{~K}$ and $\log g=4.4$ dex to the Sun, both with solar metallicity. The EWs of the same lines were measured on both real and synthetic spectra and the ratio $\mathrm{EW}$ (synthetic)/EW(real) was analysed.

We found that the EW(synthetic)/EW(real) ratio does not depend on wavelength, line strength, and excitation potential of low level of the transition. Other atomic parameters, like excitation potential of the upper level and total excitation energy, have also been tested and no dependence with $\mathrm{EW}$ (synthetic)/EW(real) ratio was found. The line-to-line scatter of this ratio can be quantified with a standard deviation from the average. We found that the sample standard deviation is about $0.2-0.3$ dex for the weakest lines with $E W<50 \mathrm{~m} \AA$ and $0.1-0.2$ dex for stronger lines. There are two possible causes for the dispersion of values: one is due to the error in measurement and determination of continuum level, and another is due to the lack of correction for the $\log g f$ and Van der Waals damping parameters in the synthetic templates. Although the line-to-line scatter of the $\mathrm{EW}$ (synthetic)/EW(real) ratio is relatively large, it does not affect our results due to the large number of lines used in the analysis.

\section{4. $\mathrm{LkH} \alpha 321$}

Two of our objects, $\mathrm{LkH} \alpha 321$ and V1331 Cyg, are located in a dark cloud in Cygnus, a few degrees north from the star forming region NGC7000/IC5070. The distance to these two objects was previously estimated as 700 pc (Chavarria 1981) but later revised to $550 \pm 50 \mathrm{pc}$ (Shevchenko et al. 1991).

The star LkH $\alpha 321$ permanently shows strong wind features in the P Cyg profiles of the Balmer lines. The [S II] images show a knotty jet extending 22 arcsec (Mundt \& Eislöffel 1998). The forbidden line of [O I] $6300 \AA$ has the central peak and the blue-shifted emission component at a radial velocity of about $-376 \mathrm{~km} \mathrm{~s}^{-1}$ with respect to the star.

This star shows low photometric variability, within visual magnitude $V=12.25-12.44$ (Grankin et al. 2007). To date, no period of axial rotation has been reported. According to Cohen \& Kuhi (1979), this star has spectral type G1 and an interstellar extinction $A_{V}=2.23 \pm 0.3 \mathrm{mag}$. The stellar bolometric luminosity estimated from these photometric data, assuming a distance $d=550 \pm 50$ pc, is: $\log \left(L_{*} / L_{\odot}\right)=1.42 \pm 0.12$.

Figures 3 and 4 show fragments of the spectrum of $\mathrm{LkH} \alpha$ 321 , including emission and photospheric absorption lines. The

3 Source: http://www.blancocuaresma.com/s/benchmarkstars

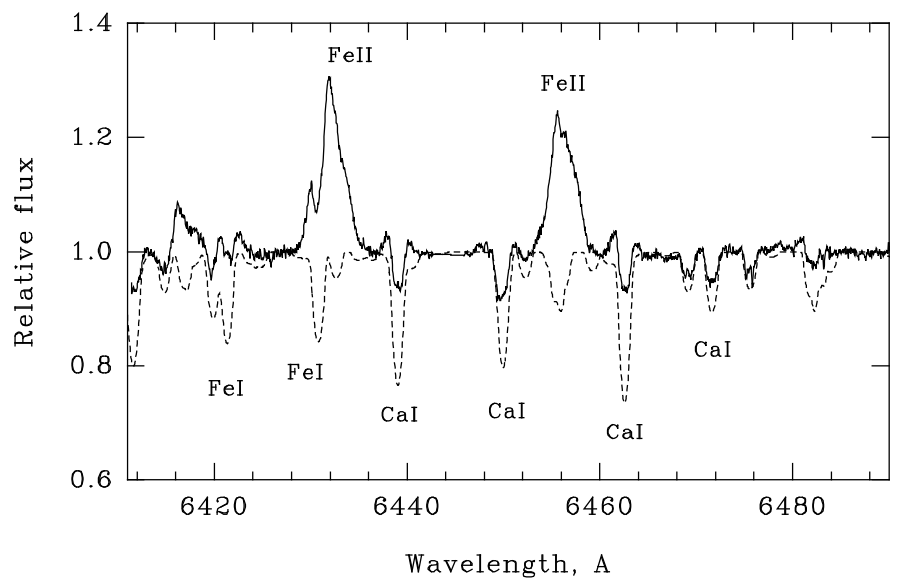

Fig. 3. Fragment of spectra of $\mathrm{LkH} \alpha 321$ (solid line) and the synthetic template $T_{\text {eff }}=5250 \mathrm{~K}, \log g=3.75$ dex, $v \sin i=32 \mathrm{~km} \mathrm{~s}^{-1}$ (dotted line). The two broad emissions belong to Fe II. The weak broad emission wings can be noticed in the strongest $\mathrm{Ca}$ I lines.

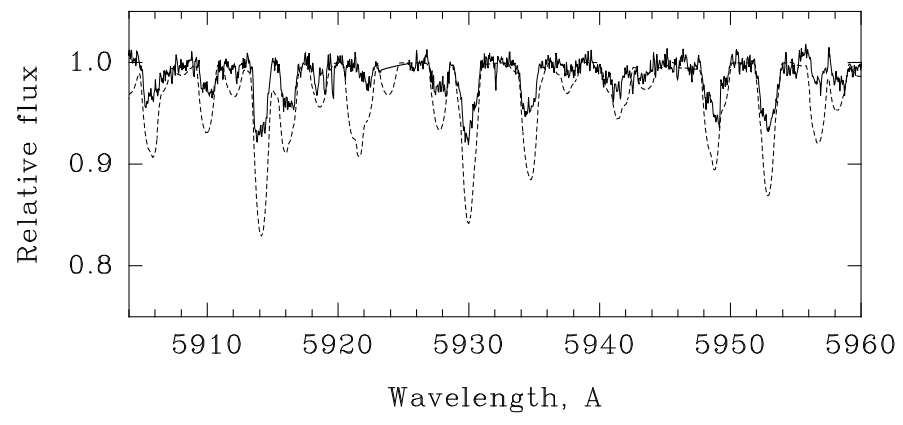

Fig. 4. Comparison of the photospheric lines in $\mathrm{LkH} \alpha 321$ and in the synthetic template. Most of the lines are of Fe I. No broad emissions in this region, but the photospheric lines are veiled considerably.

emission component of neutral metals is broader than the photospheric absorptions and becomes more noticeable in relatively strong transitions, where the photospheric counterparts have $E W \geq 100 \mathrm{~m} \AA$. In even stronger lines the broad emission is well above continuum, with the photospheric absorption on top of it. The emission lines of ions have no photospheric counterpart and display a triangular profile.

The comparison of the $\mathrm{LkH} \alpha 321$ spectrum with the grid of synthetic templates results in the best fit to $T_{\text {eff }}=5250 \mathrm{~K}$ and $v \sin i=32 \mathrm{~km} \mathrm{~s}^{-1}$ as reported in Table 3. No significant change was observed between the radial velocities measured in UES spectra, $v_{\text {rad }}=-14.4 \pm 1.0 \mathrm{~km} \mathrm{~s}^{-1}$ and in the HIRES spectra (Table 1).

After the construction of the synthetic template we were able to measure EWs of photospheric lines on both $\mathrm{LkH} \alpha 321$ and template spectrum, as described in Sect. 3.3. We investigate the dependence of the ratio $\mathrm{EW}$ (template)/EW(tts) on the line strength, wavelength, and excitation potential of the lower level of the transition.

Figure 5 shows that the amount of veiling in a photospheric line is dependent on the strength of the line in the template spectrum (corresponding to a spectral type G8). The dependence remains even if we adopt an earlier spectral type, G0. In this case the average level of veiling becomes lower. However, some lines in $\mathrm{LkH} \alpha 321$ are stronger than in a G0 template, which results in a false negative veiling and indicates that an inappropriate template star has been chosen. On the other hand, the 

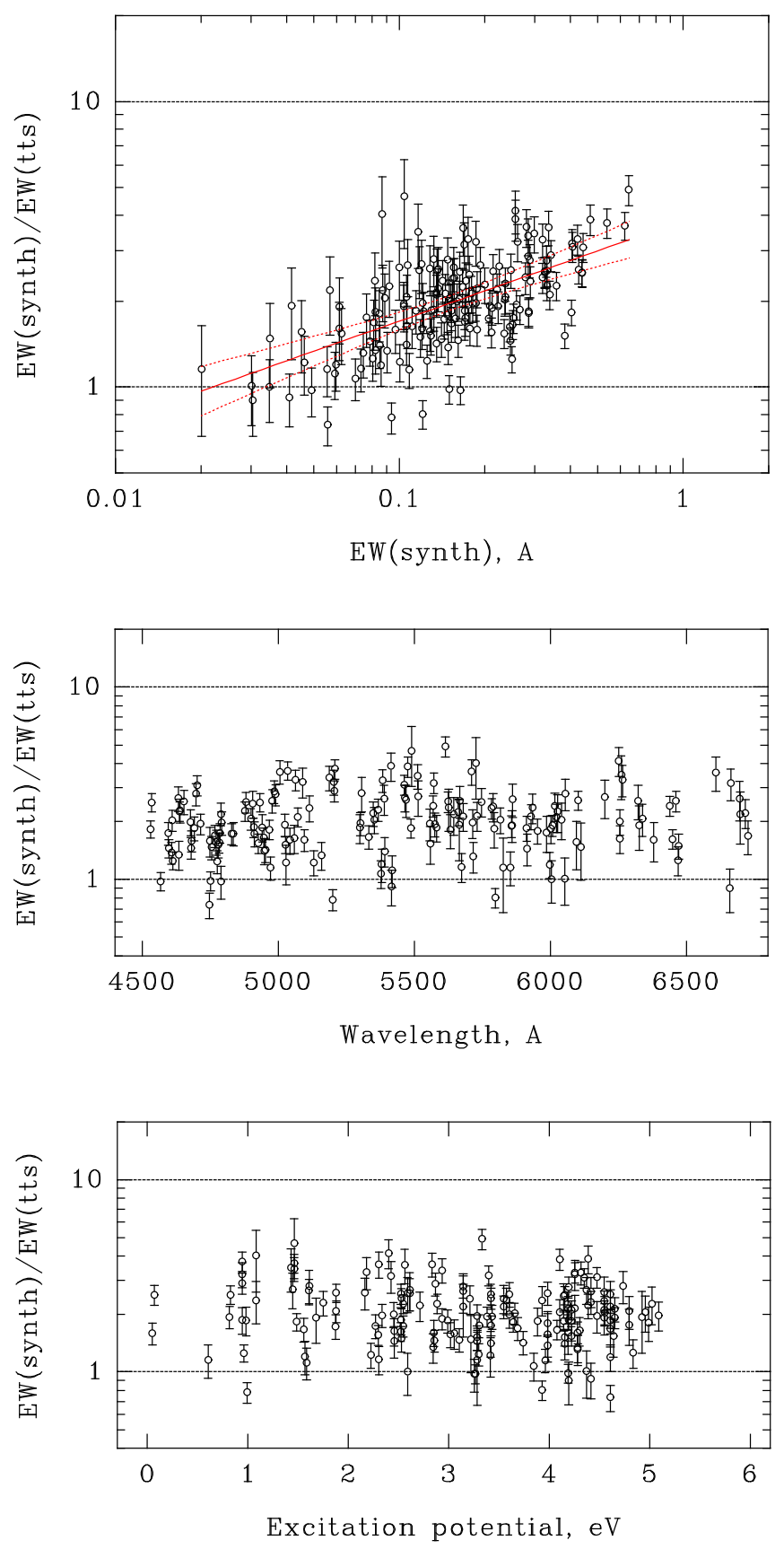

Fig. 5. Comparison of $\mathrm{LkH} \alpha 321$ with template $T_{\text {eff }}=5250 \mathrm{~K}$. Veiling as a function of line strength (upper), wavelength (middle), and excitation potential (lower). The line of linear regression and the $99 \%$ confidence interval are indicated on the top panel.

lower limit of the spectral type is $\mathrm{K} 1$, because the low-excitation $(0-1 \mathrm{eV})$ lines of neutrals are absent in the observed spectrum. Therefore, the dependence of veiling on line strength, shown in Fig. 5, is quite robust against any inappropriate spectral type determination.

The main result is that the strongest transitions are most affected by the veiling. We do not find any veiling dependence either on excitation potential or wavelength (Fig. 5). In this spectrum, the broad emission lines are unlikely to cause the line-dependent veiling. The weak broad emission can be noticed starting from EW of about $100 \mathrm{~m} \AA$ in the template spectrum, while the veiling is already strong in these lines. There must be another cause of the veiling. Although the photospheric line width is relatively large $\left(v \sin i=32 \mathrm{~km} \mathrm{~s}^{-1}\right)$, we do not see narrow emission cores there, like those observed in S CrA SE (Gahm et al. 2008) and modelled by Dodin \& Lamzin (2012).

\section{V1331 Cyg}

The unusually strong emission and wind features in V1331 Cyg make it similar to the pre-outburst spectrum of the FU Orionis type star (FUor) V1057 Cyg. For that reason, V1331 Cyg was considered as a possible pre-outburst FUor (Welin 1976). The star is viewed pole-on with a collimated outflow (jet) towards the observer (Mundt 1984; Mundt \& Eislöffel 1998). Furthermore, the star is surrounded by ring-like nebulae, which might be made up of remnants of powerful mass-loss events in the past (Kuhi 1964; Mundt \& Eislöffel 1998; Quanz et al. 2007; Choudhary et al. 2016).

The Keck spectra of V1331 Cyg, used in our research, was previously analysed by Petrov \& Babina (2014) and Petrov et al. (2014), where the presence of the line-dependent veiling was demonstrated. In addition, the spectral type of G7-K0IV, $\log g \sim$ $3.5 \mathrm{dex}$, radial velocity $v_{\mathrm{rad}}=-15.0 \mathrm{~km} \mathrm{~s}^{-1}$ and a small $v \sin i<$ $6 \mathrm{~km} \mathrm{~s}^{-1}$ were derived from the photospheric lines. From the analysis of the forbidden line profiles and the blue-shifted "shell" components of strong permitted lines, it was proved that the star is viewed through the jet (Petrov et al. 2014). The forbidden line of [O I] $6300 \AA$ has a strong central peak and a blue-shifted one at $-235 \mathrm{~km} \mathrm{~s}^{-1}$.

The star varies in brightness within $V=11.7-12.5$ (Kolotilov 1983; Shevchenko et al. 1991) and to the best of our knowledge no period related with rotation has been reported. Hamann \& Persson (1992) estimated a stellar bolometric luminosity of $L=$ $21 L_{\odot}$, with interstellar extinction $A_{V}=1.4 \mathrm{mag}$. The two spectra of 2004 and 2007 show no difference in strength of photosphere lines. The radial velocity of the star is the same in both spectra and slightly smaller in the UES spectra obtained in $1998, v_{\mathrm{rad}}=$ $-16.8 \pm 0.3 \mathrm{~km} \mathrm{~s}^{-1}$.

Spectral type analysis for V1331 Cyg places this star between the synthetic spectra with $T_{\text {eff }}=5250$ and $5000 \mathrm{~K}$, making it a G7IV-K1IV star (Table 1). This result is consistent with previous determinations by Petrov \& Babina (2014). The complete set of parameters for the V1331 Cyg synthetic template are listed in Table 3.

In Fig. 6 we present the dependence of the ratio EW(template)/EW(tts) on the line strength, wavelength, and excitation potential of the lower level of the transition for V1331 Cyg. The top panel in Fig. 6 is similar to that obtained by Petrov et al. (2014), where spectra of real stars were used as templates. Also, there is no dependence of the ratio EW(template)/EW(tts) on wavelength or excitation potential. The gaps along the wavelength scale on the middle panel of Fig. 6 reflect the regions of intense emission lines and gaps between spectral orders.

\section{AS 353A}

The star AS 353A belongs to the Aquila star forming region. The distance is estimated as $d=150 \pm 50 \mathrm{pc}$ (Prato et al. 2003; Rice et al. 2006). It is a mixed system with a primary cTTS, AS 353A, and a secondary wTTS, AS 353B. The secondary, located at 5.6" of the primary, is resolved into a subarcsecond binary, AS 353Ba and Bb, separated by $0.24 "$ (Tokunaga et al. 2004).

The star AS 353A has a rich emission line spectrum with strong blue-shifted absorptions in all the Balmer lines, indicating a powerful outflow (Herbig \& Jones 1983; Eisloeffel et al. 1990; Hamann \& Persson 1992; Alencar \& Basri 2000). The star 


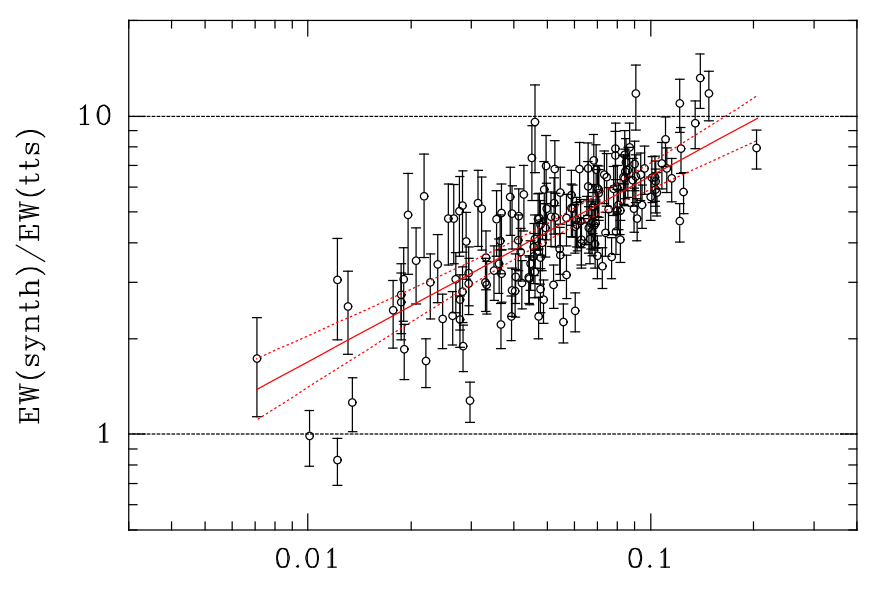

EW(synth), A
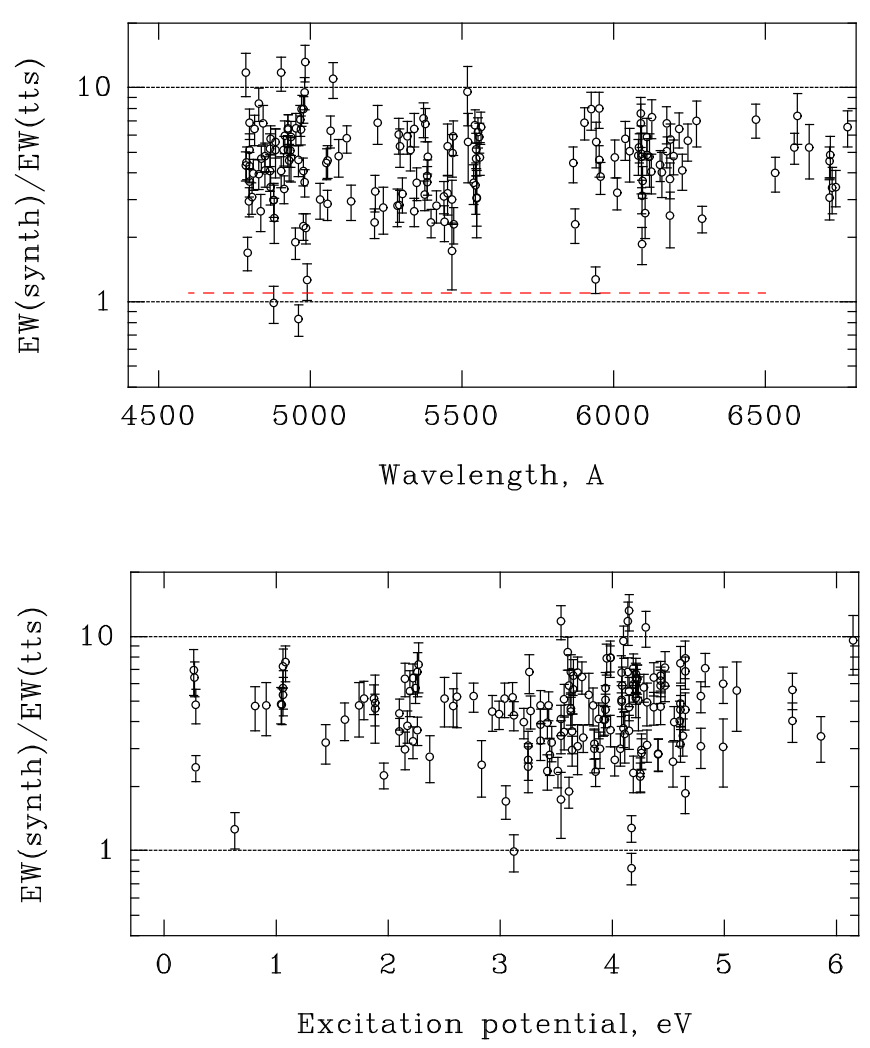

Fig. 6. Comparison of V1331 Cyg with template $T_{\text {eff }}=5000 \mathrm{~K}$. Veiling as a function of line strength (upper), wavelength (middle), and excitation potential (lower). The line of linear regression and the $99 \%$ confidence interval are indicated on the top panel. Approximate level of the veiling continuum is indicated with the dashed line in the middle panel.

drives the Herbig-Haro object HH-32 (Herbig \& Jones 1983). The forbidden line of [O I] $6300 \mathrm{~A}$ has the central peak and two components at -270 and $+250 \mathrm{~km} \mathrm{~s}^{-1}$.

Basri \& Batalha (1990) estimated the spectral type K2 from the optical spectroscopy. This was later confirmed from the $K$ band spectroscopy (Tokunaga et al. 2004). From the $H$-band spectroscopy, Rice et al. (2006) derived the following parameters for AS 353A: spectral type K5, $v \sin i=10 \mathrm{~km} \mathrm{~s}^{-1}$, and stellar radial velocity $v_{\text {rad }}=-11.4 \pm 1.1 \mathrm{~km} \mathrm{~s}^{-1}$. The stellar bolometric luminosity is $\log \left(L_{*} / L_{\odot}\right)=0.4$ (Tokunaga et al. 2004). Photometric variability within 0.86 mag in $V$ was observed by Fernandez \& Eiroa (1996). No periodicity related to stellar rotation was reported.

Our spectral type analysis placed AS 353A between the synthetic templates with $T_{\text {eff }}=4900$ and $5100 \mathrm{~K}$, corresponding to a K0IV-K1IV star. The complete information about the AS 353A synthetic template can be found in Table 3. From the HIRES spectra we measured similar radial velocity to that given by Rice et al. (2006) (see Table 1), but in the UES spectra we found higher velocity, $v_{\text {rad }}=-8.0 \pm 0.2 \mathrm{~km} \mathrm{~s}^{-1}$. This star also shows dependence of the ratio $\mathrm{EW}$ (template)/EW(tts) on the line strength (Fig. 7). Again, we found no correlation between the ratio EW(template)/EW(tts) with wavelength and excitation potential of the lower level of the transition. The spectrum of AS 353A is remarkable in the combination of the narrow photospheric lines, $v \sin i<6 \mathrm{~km} \mathrm{~s}^{-1}$, and the broad emissions of metals, $F W H M \sim 150 \mathrm{~km} \mathrm{~s}^{-1}$ (Fig. 9). This makes clear the presence of the photospheric lines on top of the broad emissions.

\section{Discussion}

Three possible sources of veiling were considered in the literature: (1) the veiling continuum radiated by a hot $\operatorname{spot}(\mathrm{s})$ at the base of accretion funnel(s) (e.g. Basri \& Batalha 1990), (2) narrow emission lines originating from the post-shocked gas (Dodin \& Lamzin 2012), and (3) broad emission lines originating in the infalling pre-shocked gas within the magnetosphere (Sicilia-Aguilar et al. 2015).

In all of our objects the different spectral lines show different veiling, with a clear dependence of veiling on the strength of transition. This cannot be explained entirely with the traditional model of a hot spot radiating the veiling continuum. The three cTTS discussed here are outstanding due to their high luminosity and high accretion rates, therefore the origin of the veiling may not be necessarily the same as in a moderately accreting cTTS. In the following we consider in more detail the possible sources of veiling.

\subsection{Broad emission}

Line-dependent veiling was investigated by Sicilia-Aguilar et al. (2015) from the spectral analysis of the EXor EX Lup in quiescence time. The authors suggested that broad emission lines, originating from the pre-shocked material, can fill in the photospheric absorptions, thus resulting in line-dependent veiling. We examined whether this interpretation may be applied for our three stars.

In AS 353A and $\mathrm{LkH} \alpha$ 321, the broad emission lines of metals are much broader than the corresponding photospheric lines (see Figs. 8 and 9). The photospheric counterparts of the broad emissions of neutral metals (mostly Fe I) are well seen on top of the emissions and are reduced in depth by about the same factor as the other strong photospheric lines without the broad emission component. It means that the broad emission component does not contribute considerably to the line-dependent veiling. The observed profile is just a sum of the optically thin broad emission and the underlying photospheric absorption. The only way the broad emission can affect the photospheric lines is that blends of the broad emissions may form an additional local pseudo continuum. In our analysis of the veiled photospheric lines, we avoided those lines which might be contaminated by such blends.

The third of our objects, V1331 Cyg, is remarkable for its pole-on orientation towards the observer. The low excitation emission lines of neutral and ionized metals appear at stellar 

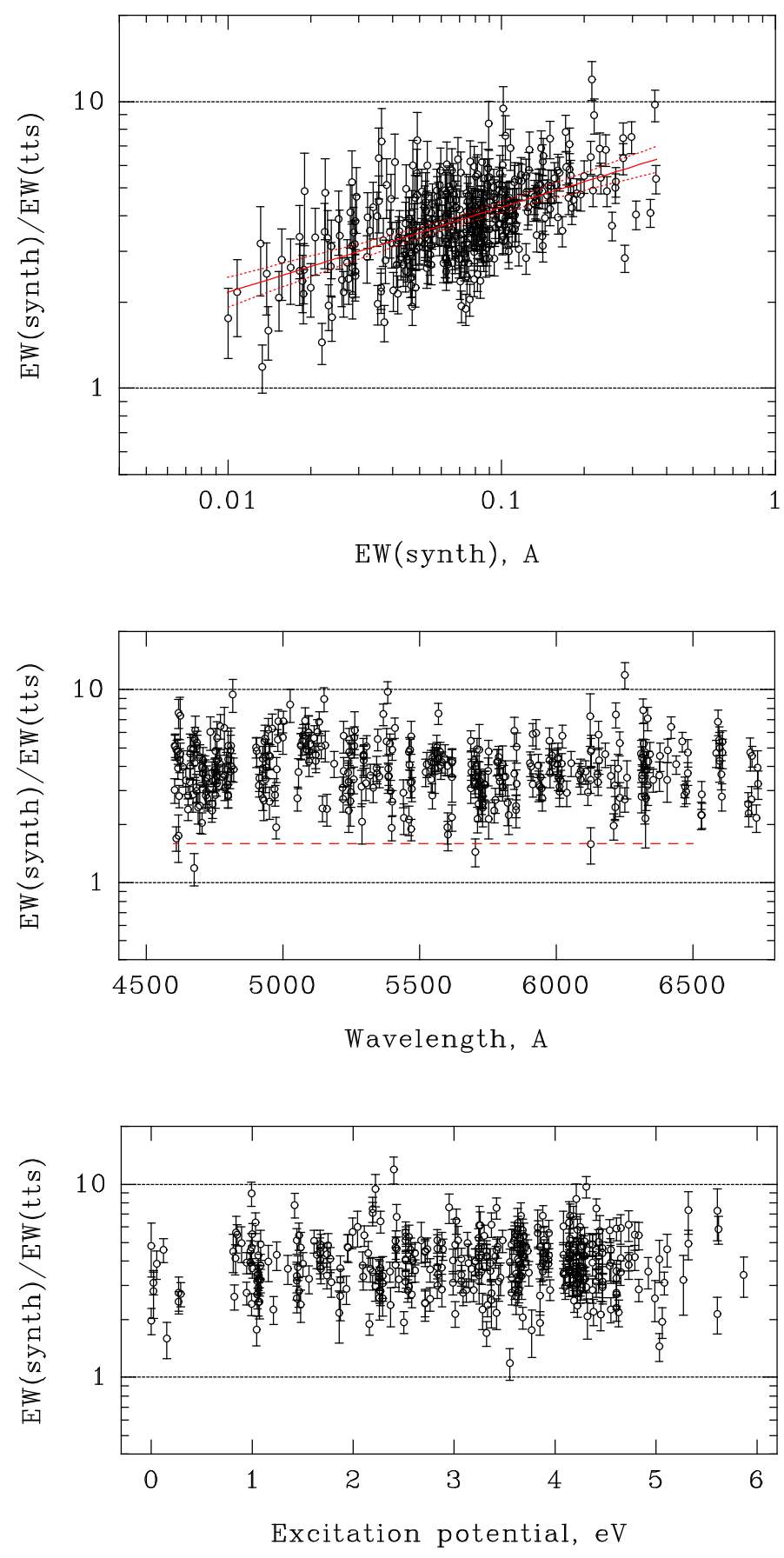

Fig. 7. Comparison of AS 353A with template $T_{\text {eff }}=5000 \mathrm{~K}$. Veiling as a function of line strength (upper), wavelength (middle), and excitation potential (lower). The line of linear regression and the $99 \%$ confidence interval are indicated on the top panel. Approximate level of the veiling continuum is indicated with the dashed line in the middle panel.

velocity, and look similar in the three spectra taken in 2002, 2004, and 2007 (Fig. 10). The emission lines appear as not broadened by the flows within the stellar magnetosphere, probably because of the pole-on orientation of the star.

\subsection{Photospheric hot spot}

Our results, presented above, show that the veiling continuum can be quantified only by the weak photospheric lines. Measuring the weakest lines, we probed the deepest layers

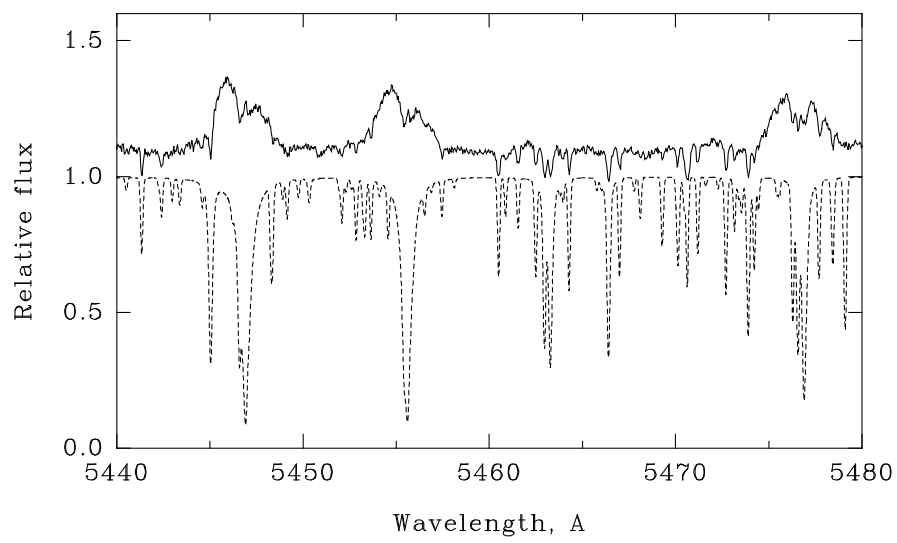

Fig. 8. Spectral fragments of AS 353A and the corresponding synthetic template (dashed line). We note the photospheric absorption lines on top of the broad emissions of Fe I.

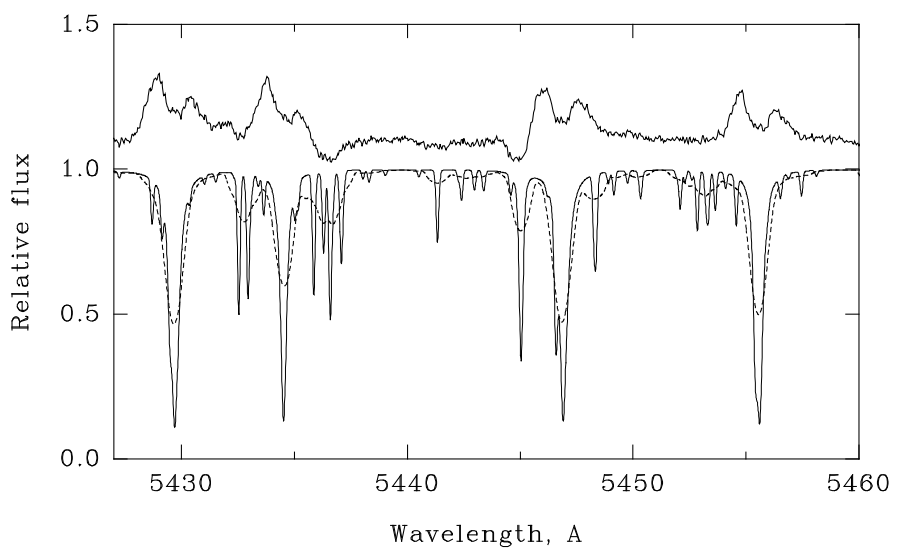

Fig. 9. Spectral fragments of $\mathrm{LkH} \alpha 321$ and the corresponding synthetic template (thin line). The template broadened to $v \sin i=32 \mathrm{~km} \mathrm{~s}^{-1}$ is overplotted with dashed line. We note the photospheric absorption lines on top of the broad emissions of Fe I.

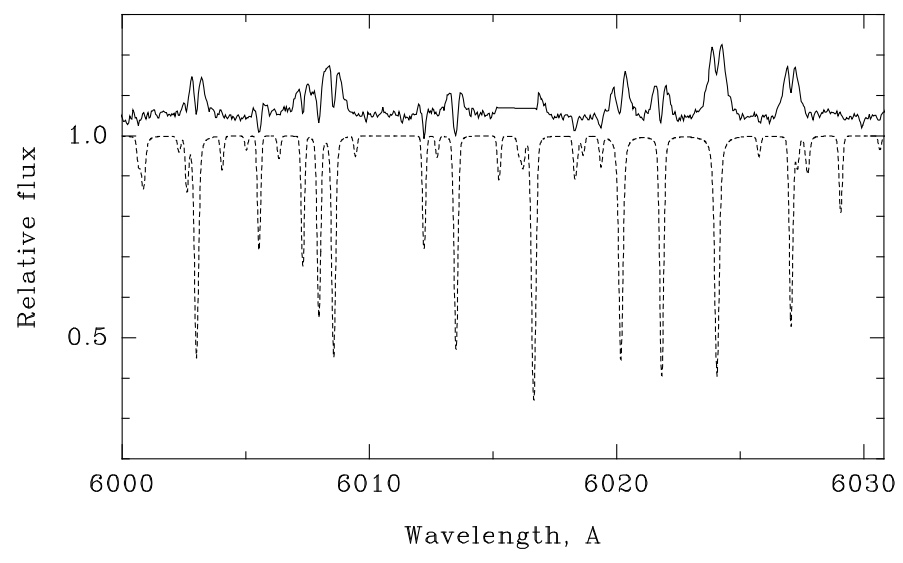

Fig. 10. Spectral fragment of V1331 Cyg and the corresponding synthetic template (dashed line). We note the photospheric absorption dips on top of the emission lines of Fe I and Mn I.

of line formation. We also found that the veiling continuum determination is affected by the spectral resolution and $\mathrm{S} / \mathrm{N}$ level. The spectra with lower resolution and/or poor $\mathrm{S} / \mathrm{N}$ ratio lead to an overestimation of the veiling continuum.

The hot spot hypothesis assumes that the accretion luminosity and the veiling continuum can be explained as being due to 
radiation of a hot spot, with temperature $T_{\text {spot }}$, and a filling factor, $f$, representing the relative size of hot spot region with respect to the stellar surface. The accretion luminosity can be estimated from the observed emission lines. It has been shown that $L_{\text {acc }}$ is well correlated with the line luminosity, $L_{\text {line }}$, of several emission lines, though the uncertainties in the empirical relations are in some cases high (e.g. Fang et al. 2009; Rigliaco et al. 2012; Alcalá et al. 2014 and references therein). In our study we opted for the empirical relations $L_{\mathrm{acc}}-L_{\text {line }}$ obtained by Alcalá et al. (2014), which span a larger range in mass. In our sample of very active cTTS, the $\mathrm{H} \alpha$ and $\mathrm{H} \beta$ emissions are not good indicators because of the large optical depth and strong P Cygni profiles, which remove the blue emission wings of those lines (as seen in Fig. 1). Therefore, we assume that the He I $5876 \AA$ line is more reliable to estimate the accretion luminosity.

We first determine the ratio $L_{\text {line }} / L_{\text {star }}$ through the equation

$\frac{L_{\text {line }}}{L_{\text {star }}}=E W *(1+V C) * \frac{F_{\text {cont }}}{L_{\text {star }}}$

where $L_{\text {star }}$ is the stellar bolometric luminosity, $F_{\text {cont }}$ is the stellar continuum flux density at the wavelength of the He I line, EW is the equivalent width of the $\mathrm{He}$ I emission, and $\mathrm{VC}$ is the veiling continuum near the $\mathrm{He}$ I line.

The ratio $F_{\text {cont }} / L_{\text {star }}$ is obtained using the adequate template spectra selected from the stellar spectral flux library by Pickles (1998). The library covers the spectral range 1150$25000 \AA$, and a blackbody at the star temperature is added to simulate the continuum level at wavelengths longer than $2.5 \mu \mathrm{m}$ for a better determination of $L_{\mathrm{star}}$.

The ratio $L_{\text {line }} / L_{\text {star }}$ is converted to $L_{\text {acc }} / L_{\text {star }}$ using the relationship obtained by Alcalá et al. (2014) and the mass accretion rate determined from the equation

$\dot{M}_{\mathrm{acc}}=\frac{L_{\mathrm{acc}} R_{*}}{G M_{*}}\left(1-R_{*} / R_{\mathrm{in}}\right)^{-1}$,

where $R_{*}$ and $M_{*}$ are the star radius and mass, respectively, reported in Table 1 , and $R_{\text {in }}$ is the inner disk radius taken as $3 R_{*}$ in our calculation. Table 4 displays the final results, including the veiling continuum estimated from the plots EW/EW versus wavelength, the He I line, and accretion luminosities used for the mass accretion rate determination.

We have also checked that the accretion luminosities and the veiling continuum reported in Table 4 are consistent with the hot spot hypothesis. Within the used spectral range (4350 $6750 \AA$ ), we do not see dependence of the veiling continuum on wavelength, which makes the estimation of the hot spot(s) temperature more difficult. We may only assert that temperatures higher than $10000 \mathrm{~K}$ for the spots are not expected, otherwise the slope in the plots EW/EW versus wavelength would be more pronounced.

If we consider the typical spot temperatures for cTTS in the range 6000-8000 K (Calvet \& Gullbring 1998) and assuming blackbody emission from the spot, we may infer the spot filling factor in our stars by using the equation:

$\frac{L_{\mathrm{acc}}}{L_{\mathrm{star}}} \sim f\left(\frac{T_{\mathrm{spot}}}{T_{\mathrm{star}}}\right)^{4}$,

where $T_{\text {star }}$ is the effective temperature of the star.
We get filling factor values between $1 \%$ and $3 \%$ for V1331 Cyg 7, 20\% for AS 353A, and below $1 \%$ for $\mathrm{LkH} \alpha$ 321. The filling factor for AS 353A seems to be unusually large, which may be caused by an overestimated veiling continuum for this star.

\subsection{Accretion-powered chromosphere}

The dependence of veiling on the strength of transition indicates that the origin of this veiling is related to a drastic change in the stellar atmosphere structure due to accretion. The upper layers of the stellar atmosphere are most affected by the hard radiation of the shock wave located above. Therefore, at a given accreting mass flux the cores of strong lines will be mostly affected, while the weakest lines formed in the deeper layers remain still unchanged. In this situation, only the weakest lines may be used as a measure of the veiling continuum.

Dodin \& Lamzin (2012) considered a model of veiling by lines, where the narrow emission lines, radiated in the post-shock region, contribute to the veiling along with the continuum radiation from the hot spot. The model showed that the veiling by lines prevails in cTTS with a moderate accretion flux, while with increasing accretion flux the veiling continuum becomes the most important. Our results do not contradict this prediction: the relative contribution of the veiling continuum is greater in AS 353A, where $L_{\text {acc }} / L_{\text {star }}$ is the largest of the three stars.

If the line-dependent veiling is caused by the emission lines radiated in a small post-shock region, which is hotter than the surrounding undisturbed photosphere, one would expect an emission core in the strongest lines and/or a dependence of veiling on the excitation potential. None of our objects reveals the dependence of veiling on excitation potential, probably because of the large scatter of points on the diagrams.

In AS 353A and V1331 Cyg, the $v \sin i$ is too small to resolve the details in a photospheric line profile. However, in the case of $\mathrm{LkH} \alpha 321$, the $v \sin i$ is larger and we can conclude from the absence of a narrow emission core in relatively broad photospheric lines that the line emission is not concentrated in a single spot but spread over a larger area above the visible stellar surface. A time series of spectral observations of this star would reveal probable variations in the photospheric line profiles. The mapping of magnetic field, cool spots, and emission line areas on the stellar surface was done for several cTTS (see Donati et al. 2013; Johnstone et al. 2014, and references therein). In all cases there is an axial asymmetry in the distribution of the emission line areas.

In $\mathrm{LkH} \alpha 321$ the line width is large enough to apply Doppler imaging. In V1331 Cyg and AS 353A, where the photospheric lines are so narrow, it is still possible to look for variations in radial velocity caused by a passage of the presumable hot spot(s) across the visible stellar surface. So far we can only assert that there is no change in the radial velocity of V1331 Cyg within $\pm 0.3 \mathrm{~km} \mathrm{~s}^{-1}$ as derived from the HIRES spectra of 2004 and 2007. A significant change in radial velocity was noticed only in AS 353A: $v_{\text {rad }}=-8.0 \pm 0.2 \mathrm{~km} \mathrm{~s}^{-1}$ in 1998 and $-10.4 \pm 0.2 \mathrm{~km} \mathrm{~s}^{-1}$ in 2004 .

The most intriguing is that of $\mathrm{LkH} \alpha 321$, where the veiling continuum is absent, but the line-dependent veiling is large. A similar effect was observed in DR Tau (Petrov et al. 2011). In that star both the veiling continuum and the line-dependent veiling vary from night to night. When the veiling continuum disappears, the line-dependent veiling remains, although at a reduced level. It was interpreted as an accretion-powered chromosphere. At a given infall velocity, the accretion energy flux is proportional to the density of infalling gas. At lower density the 
Table 4. Determination of accretion luminosities and mass accretion rates.

\begin{tabular}{cccccc}
\hline \hline Star & Template & VC & $\begin{array}{c}\log \left(L_{\mathrm{He}} / L_{\text {star }}\right) \\
(\mathrm{dex})\end{array}$ & $\begin{array}{c}\log \left(L_{\text {acc }} / L_{\text {star }}\right) \\
(\mathrm{dex})\end{array}$ & $\begin{array}{c}\log \left(\dot{M}_{\mathrm{acc}}\right) \\
\left(M_{\odot} \mathrm{yr}^{-1}\right)\end{array}$ \\
\hline LkH $\alpha$ 321 & G8V & 0.0 & -4.97 & -1.72 & -7.4 \\
V1331 Cyg & K0V & 0.1 & -4.51 & -1.21 & -6.9 \\
AS 353A & K0V & 0.6 & -3.6 & -0.31 & -7.1 \\
\hline
\end{tabular}

Notes. VC is the veiling continuum at the wavelength of the He I line.

photosphere is not affected, while the upper layers of atmosphere are heated, thus giving rise to chromospheric-like emissions filling in the photospheric absorptions.

Yet one more consideration concerns the magnetic field structure. The Zeeman Doppler Imaging technique enables us to restore the large-scale topology of the stellar magnetic field in cTTS (e.g. Johnstone et al. 2014). So far, the Sun is the only star for which the small-scale (down to megameters) structures of magnetic fields have been investigated. It was found that the solar magnetic field has a fractal (scale-invariant) structure (Abramenko 2008). In fact, we expect that the local magnetic fields in cTTS within any small fraction of stellar surface may be as complex as in the Sun. In the framework of the magnetospheric accretion model, the shock front is supposed to be a confined area over a small fraction of the stellar surface. One can imagine that as the ionized infalling gas approaches the star, it meets more complicated magnetic structures and finally enters the stellar atmosphere at the dividing lines between the local closed fields. How would this alter the shock structure and the heating of the underlying photosphere? These small-scale processes may be important in the manifestation of the observed consequences of accretion.

\section{Conclusions}

The analysis of spectra of three very active cTTS of low to intermediate mass show that veiling consists of two components: a line-dependent veiling and the veiling continuum. Those two components are visible when the ratios of equivalent widths of nearby lines between template and TTS are plotted against the line strength on the template. Stronger lines are more veiled than weaker lines, regardless of wavelength and excitation potential. The veiling continuum was determined from the ratio of the weakest lines and the accretion luminosities from the $\mathrm{He}$ I line emission. In the three studied stars, we found that the photospheric line veiling is dominated by the line-dependent component, while the veiling continuum is a minor contributor. We conclude that the observed veiling on the photospheric lines of $\mathrm{LkH} \alpha$ 321, AS 353A, and V1331 Cyg is composed of a line-dependent veiling with its origin in an abnormal structure of stellar atmosphere being heated up by the accreting matter and a veiling continuum radiated by a hot spot with temperature lower than $10000 \mathrm{~K}$.

Acknowledgements. This work was supported by Fundação para a Ciência e a Tecnologia (FCT) through national funds (UID/FIS/04434/2013) and by FEDER through COMPETE2020 (POCI-01-0145-FEDER-007672). A.C.S.R. acknowledges the support of an IA fellowship: CIAAUP-17/2015-BI in the context of the project (UID/FIS/04434/2013\&POCI-01-0145-FEDER-007672). P.P.P. acknowledges the visitor's programme grant from Instituto de Astrofisica e Ciências do Espaço, Portugal. This work has made use of the VALD database, operated at Uppsala University, the Institute of Astronomy RAS in Moscow, and the University of Vienna.

\section{References}

Abramenko, V. I. 2008, in Solar Physics Research Trends, ed. P. Wang (New York: Nova Publishers), 95

Alcalá, J. M., Natta, A., Manara, C. F., et al. 2014, A\&A, 561, A2

Alencar, S. H. P., \& Basri, G. 2000, AJ, 119, 1881

Basri, G., \& Batalha, C. 1990, ApJ, 363, 654

Beristain, G., Edwards, S., \& Kwan, J. 1998, ApJ, 499, 828

Blanco-Cuaresma, S., Soubiran, C., Jofré, P., \& Heiter, U. 2014, A\&A, 566, A98

Calvet, N., \& Gullbring, E. 1998, ApJ, 509, 802

Calvet, N., Muzerolle, J., Briceño, C., et al. 2004, AJ, 128, 1294

Chavarria, C. 1981, A\&A, 101, 105

Choudhary, A., Stecklum, B., \& Linz, H. 2016, A\&A, 590, A106

Cohen, M., \& Kuhi, L. V. 1979, ApJ, 41, 743

Dahm, S. E. 2008, AJ, 136, 521

Dodin, A. V., \& Lamzin, S. A. 2012, Astron. Lett., 38, 649

Donati, J.-F., Gregory, S. G., Alencar, S. H. P., et al. 2013, MNRAS, 436, 881

Eisloeffel, J., Solf, J., \& Boehm, K. H. 1990, A\&A, 237, 369

Fang, M., van Boekel, R., Wang, W., et al. 2009, A\&A, 504, 461

Fernandez, M., \& Eiroa, C. 1996, A\&A, 310, 143

Gahm, G. F., Walter, F. M., Stempels, H. C., Petrov, P. P., \& Herczeg, G. J. 2008, A\&A, 482, L35

Gameiro, J. F., Folha, D. F. M., \& Petrov, P. P. 2006, A\&A, 445, 323

Grankin, K. N., Melnikov, S. Y., Bouvier, J., Herbst, W., \& Shevchenko, V. S. 2007, A\&A, 461, 183

Hamann, F., \& Persson, S. E. 1992, ApJ, 394, 628

Hartigan, P., Kenyon, S. J., Hartmann, L., et al. 1991, ApJ, 382, 617

Hartigan, P., Edwards, S., \& Ghandour, L. 1995, ApJ, 452, 736

Herbig, G. H., \& Jones, B. F. 1983, AJ, 88, 1040

Herczeg, G. J., \& Hillenbrand, L. A. 2008, ApJ, 681, 594

James, D. J., Melo, C., Santos, N. C., \& Bouvier, J. 2006, A\&A, 446, 971

Johnstone, C. P., Jardine, M., Gregory, S. G., Donati, J.-F., \& Hussain, G. 2014, MNRAS, 437, 3202

Joy, A. H. 1949, ApJ, 110, 424

Kolotilov, E. A. 1983, Sov. Astron. Lett., 9, 289

Kuhi, L. V. ApJ, 140, 1409

Mendigutía, I., Oudmaijer, R. D., Rigliaco, E., et al. 2015, MNRAS, 452, 2837

Mundt, R. 1984, ApJ, 280, 749

Mundt, R., \& Eislöffel, J. 1998, AJ, 116, 860

Muzerolle, J., Calvet, N., \& Hartmann, L. 1998, ApJ, 492, 743

Padgett, D. L. 1996, ApJ, 471, 847

Petrov, P. P., \& Babina, E. V. 2014, Bull. Crim. Astrophys. Obs., 110, 1

Petrov, P. P., Gahm, G. F., Gameiro, J. F., et al. 2001, A\&A, 369, 993

Petrov, P. P., Gahm, G. F., Stempels, H. C., Walter, F. M., \& Artemenko, S. A. 2011, A\&A, 535, A6

Petrov, P. P., Kurosawa, R., Romanova, M. M., et al. 2014, MNRAS, 442, 3643

Pickles, A. J. 1998, PASP, 110, 863

Prato, L., Greene, T. P., \& Simon, M. 2003, ApJ, 584, 853

Quanz, S. P., Apai, D., Henning, Th. 2007, ApJ, 656, 287

Rice, E. L., Prato, L., \& McLean, I. S. 2006, ApJ, 647, 432

Rigliaco, E., Natta, A., Testi, L., et al. 2012, A\&A, 548, A56

Rojas, G., Gregorio-Hetem, J., \& Hetem, A. 2008, MNRAS, 387, 1335

Ryabchikova, T., Piskunov, N., Kurucz, R. L., et al. 2015, Phys. Scr., 90, 054005

Shevchenko, V. S., Yakulov, S. D., Hambarian, V. V., \& Garibjanian, A. T. 1991, AZh, 68, 275

Sicilia-Aguilar, A., Fang, M., Roccatagliata, V., et al. 2015, A\&A, 580, A82

Siess, L., Dufour, E., \& Forestini, M. 2000, A\&A, 358, 593

Taguchi, Y., Itoh, Y., \& Mukai, T. 2009, PASJ, 61, 251

Tokunaga, A. T., Reipurth, B., Gässler, W., et al. 2004, AJ, 127, 444

Valenti, J. A., \& Fischer, D. A. 2005, ApJS, 159, 141

Valenti, J. A., \& Piskunov, N. 1996, A\&AS, 118, 595

Valenti, J. A., Basri, G., \& Johns, C. M. 1993, AJ, 106, 2024

Vogt, S. S., Allen, S. L., Bigelow, B. C., et al. 1994, Proc. SPIE, 2198, 362

Welin, G. 1976, A\&A, 49, 145 